\title{
What have we Learned about the Different COVID-19 Phenotypes in the Pediatric Population so Far?
}

\author{
Andressa Mussi Soares ${ }^{1,2}$ and Bernardo Mussi Soares ${ }^{3 \oplus}$ \\ Hospital Evangélico de Cachoeiro de Itapemirim, ${ }^{1}$ Cachoeiro de Itapemirim, ES - Brazil \\ Faculdade de Medicina da Universidade de São Paulo, ${ }^{2}$ São Paulo, SP-Brazil \\ Fundação Técnico Educacional Souza Marques, ${ }^{3}$ Rio de Janeiro, $R J$ - Brazil \\ Editorial referring to the article: Kawasaki-like disease, a new phenotype in SARS-CoV-2?
}

The understanding of SARS-CoV-2 related syndromes in the pediatric population needs to grow worldwide. In its severe presentation, COVID-19 is a systemic disease characterized by hyperinflammation, cytokine storm and increased myocardial injury markers. Cardiac involvement in seen in $20-30 \%$ of hospitalized adult patients and accounts for about $40 \%$ of deaths. ${ }^{1}$

An intriguing aspect of the COVID-19 is the fact that most children and adolescents have mild or asymptomatic forms of the disease, perhaps related to a lower expression of virus receptors, recent exposure to other coronaviruses generating cross-protection, more developed innate immunity, among others. ${ }^{2}$ In fact, it is known that $40 \%$ of individuals under the age of 18 exposed to the virus get infected and that only $20-30 \%$ of children and adolescents infected with SARS-Cov-2 have symptoms.

However, in some cases, a late immune response (up to 6 weeks after the insult) has been observed, with involvement of one or more organs (cardiac, renal, respiratory, gastrointestinal or neurological organ), due to a massive inflammatory response that was called Multisystem Inflammatory Syndrome in Children (MIS-C). The World Health Organization (WHO) defined very clear criteria for MIS-C, including clinical, laboratory and echocardiographic aspects that have helped in the diagnosis and management of children

\section{Keywords}

COVID-19; Coronavirus; Cardiomyopathies; Child; Heart Failure; Severe Acute Respiratory Syndrome; Myocardial Stunning; Kawasaki Disease. and adolescents. ${ }^{3}$ Henderson et al., ${ }^{4}$ described different phenotypes of COVID-19 in the pediatric population, and proposed a guidance document on the management of MIS-C associated with COVID-19. According to the authors, clinical manifestations of this combination are: 1- febrile syndrome: characterized by persistent fever, mild change in inflammatory markers and indirect signs of organ dysfunction; 2-Classic MIS-C: important change in inflammatory markers, multisystem involvement and severe cardiac involvement with shock and cardiovascular dysfunction; 3- Kawasaki-like disease: cutaneous-mucosal involvement with milder cardiac involvement (American Heart Association criteria for complete or incomplete Kawasaki disease); 4- COVID-19 simile: major respiratory involvement (acute respiratory distress syndrome). ${ }^{4}$

Despite the lower number of deaths in the pediatric age group than adults, higher mortality rates due to COVID-19 in children have been recorded in Brazil compared with other countries (852 deaths in children under nine years and 518 deaths in infants younger than one year of age), although a reduction in mortality from $8.2 \%$ in 2020 to $5.8 \%$ in 2021 has already been observed in the population aged 0 to 19 years. ${ }^{5}$

The first case of Kawasaki-like disease in Covid-19 was reported in a 6-month-old infant with fever, tachycardia, signs of respiratory distress, maculopapular rash, conjunctival hyperemia, who was treated with intravenous immunoglobulin (IVIG) and high-dose aspirin, with clinical improvement. Since this report, numerous cases have been reported during the pandemic. ${ }^{6}$ DeBiasi et al. ${ }^{7}$ reported 177 young patients diagnosed with COVID-19. Of these, $44(24.8 \%)$ required hospitalization, nine $(2.8 \%)$ were critically ill and one developed signs and symptoms of Kawasaki-like disease, mainly 
cardiovascular ones, including ventricular dysfunction and arterial hypotension. ${ }^{7}$ Verdoni et al., ${ }^{8}$ observed a 30-fold increased incidence of Kawasaki-like disease in a month during the pandemic compared to previous years. ${ }^{8}$ The mechanisms of Kawasaki-like disease seem to be based on the exacerbated inflammatory reaction mediated by antibodies, which occurs mainly in genetically predisposed individuals. Therefore, in most cases, the identification of viral replication is not relevant (RT-PCR may already be negative at this stage), and the disease is better diagnosed by serology (IgM, IGG or both), emphasizing that SARS$\mathrm{CoV}-2$ infection is not mandatory, and the patient may only have a history of exposure.

Some studies have drawn attention to the absence of Kawasaki-like and MIS-C cases in Asian countries where the Covid-19 pandemic started, probably due to ethnic, genetic, and immunological factors, with higher incidence of cases in populations of African ancestry.

The scientific paper "Kawasaki-like disease, a new phenotype in Sars-CoV-2?"9 brings interesting information about SARS-CoV-2 in childhood and adolescence and its association with the Kawasaki-like disease and MIS-C, ${ }^{9}$ and highlights different clinical and laboratory aspects that stand out in cases of Kawasaki-like disease. For example, a higher frequency of gastrointestinal manifestations (abdominal pain, mesenteric lymphadenopathy, vomiting, diarrhea, and in some cases an acute abdomen requiring exploratory surgery), lymphopenia, elevation of inflammatory markers and myocardial injury (C-reactive protein, interleukins, procalcitonin, D-dimer, N-terminal prohormone of brain natriuretic peptide [NT-proBNP], troponin, lactate, transaminases, etc.), cardiovascular manifestations such as myocarditis, valvulitis, left and/or right ventricular dysfunction, changes in the coronary artery and pericardium, hemodynamic instability, shock and greater need for intensive care support compared to classic Kawasaki. Table 1 lists aspects of cardiovascular involvement in MIS-C with Kawasaki-like disease and aspects of classic Kawasaki disease (Figures 1 and 2).

Fedstein et al., ${ }^{10}$ published the manifestation of MIS-C in 186 children and adolescents (mean age 8.3 years) in 26 American states; ${ }^{10} 148$ patients $(80 \%$ ) had cardiovascular manifestations, 74 had Kawasaki-like disease $(40 \%), 15$ patients ( $8 \%$ with z-score $>2.5$ ) had coronary-artery aneurysms, and four patients died (2\%). Elevation of NT-proBNP occurred in $73 \%$ and left ventricular dysfunction with $\mathrm{LVEF}<55 \%$ in $33 \%$.

Table 1 - Most common differences found in cardiovascular manifestations on echocardiography and ECG in Kawasaki-like and Kawasaki disease and in markers of inflammatory evidence and myocardial injury.

\begin{tabular}{l} 
Kawasaki-like disease \\
\hline ECG (100\%): tachycardia \\
- negative T wave, QRS with decreased amplitude \\
Echocardiogram \\
- Moderate to severe LV dysfunction \\
- Right ventricular dysfunction \\
- Valvulitis \\
- Coronary changes (increase in brightness) \\
- Pericardial effusion ( mild/moderate/important)
\end{tabular}

CRP 令令令令

Platelets $\sqrt{ }$

Ferritin 仓

D- dimer 仑̂ि仑

NT-proBNP 它苗艺

Troponin 사나
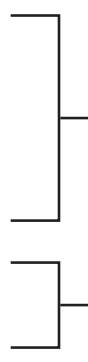

Myocardial injury
Kawasaki disease

ECG (30\%): tachycardia

- negative T wave, QRS with decreased amplitude

\author{
Echocardiogram \\ - Mild LV dysfunction \\ - Mild pericardial effusion \\ - Valvulitis \\ - Coronary changes (with dilations and aneurysms - z-scores $\hat{\imath})$
}

\section{仓ิ 仑ิ}

仓ิ

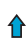

ิิ

令

ิิ

Yes

ECG: electrocardiography; LV: left ventricular; NT-proBNP: N-terminal prohormone of brain natriuretic peptide 

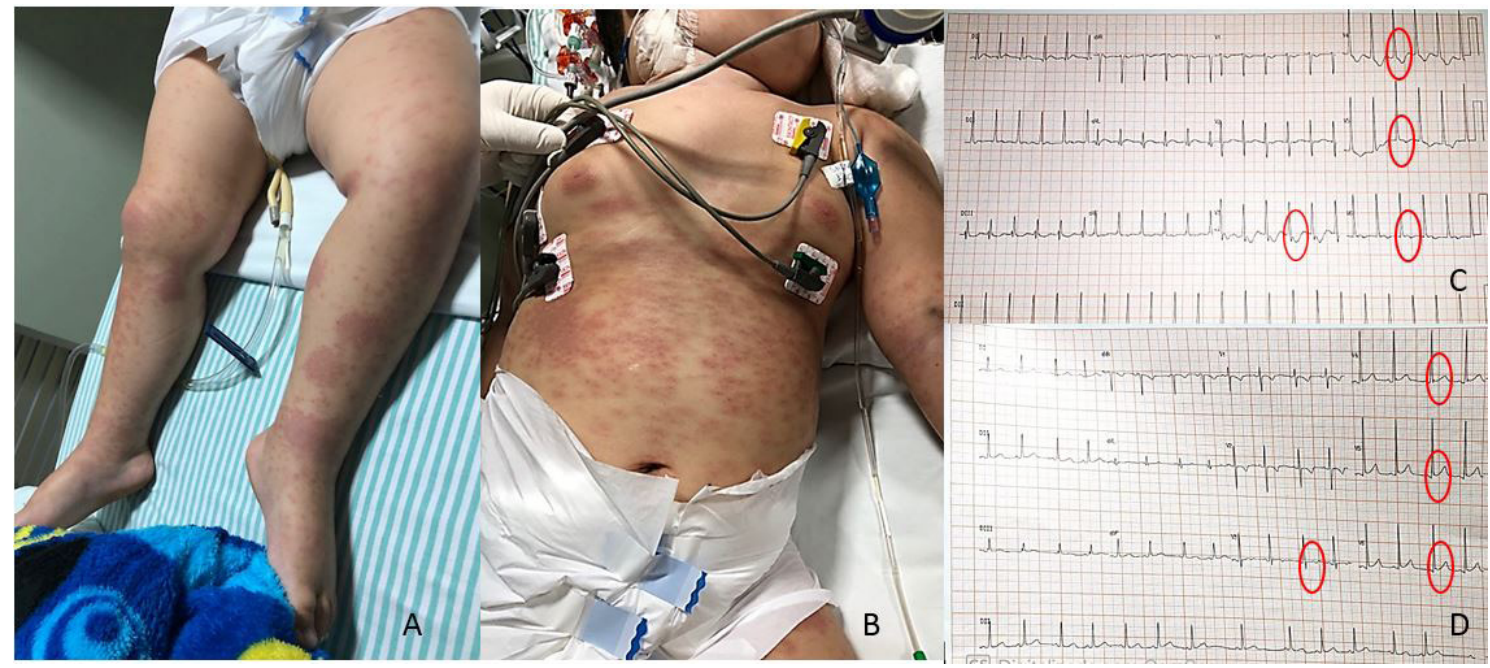

Figure 1 - Boy, 3 years old, weight: 30 kg, with Kawasaki-like, fever, skin rash (A,B), vomiting, respiratory distress and severe hemodynamic instability and myocarditis. ECG with negative T waves in the left precordials before IVIG infusion and corticosteroid (C) and already normal T waves after therapy (D).

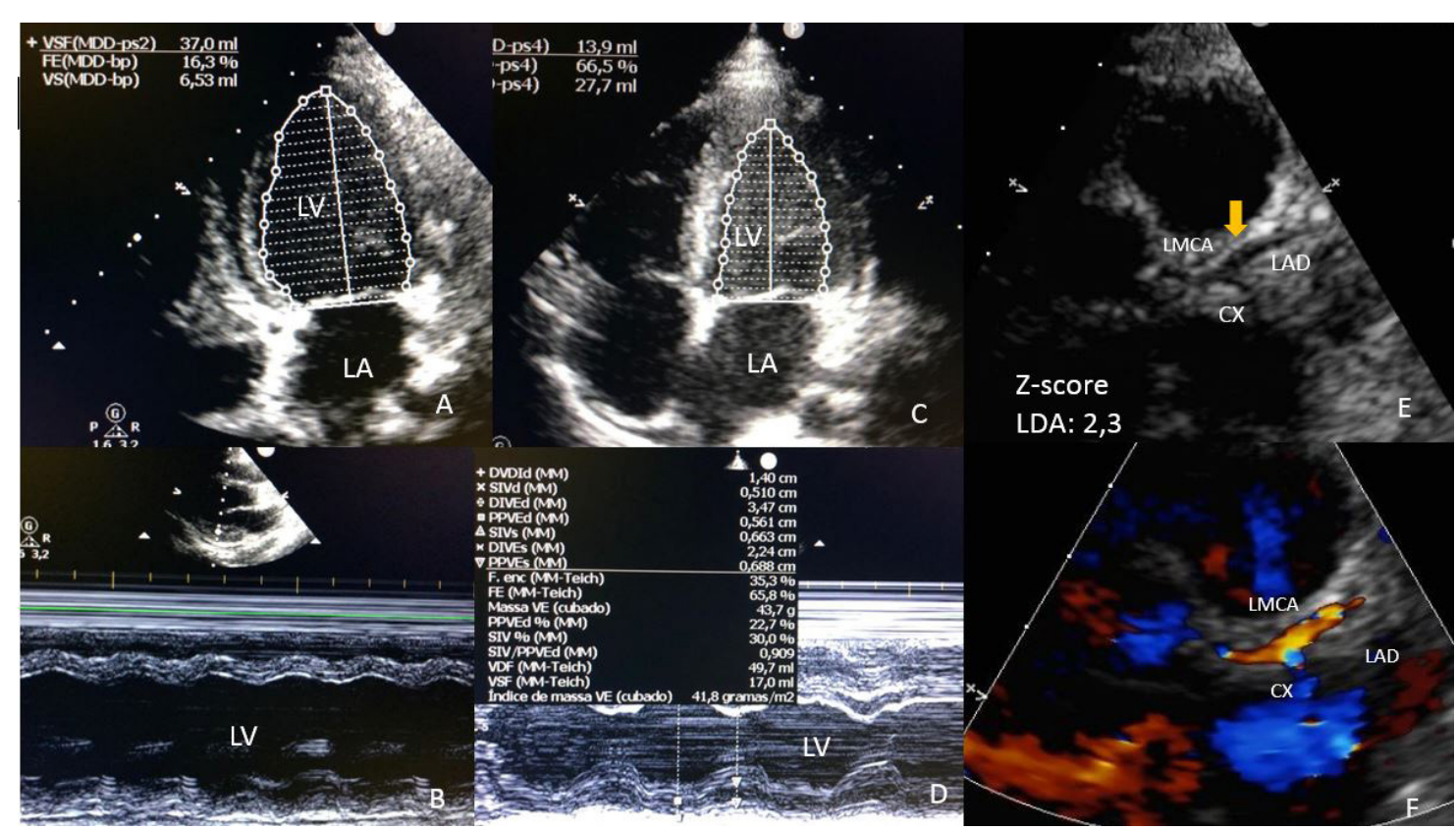

Figure 2 - Transthoracic echocardiogram of the boy with Kawasaki-like showing severe left ventricular dysfunction by Simpson (A) and Teichholz (B) and significant improvement after treatment (C,D). LDA dilation (z-score : 2,3) with increased brightness and refringence of the left coronary wall on the twodimensional (E) and on the color Doppler (F) . LV: left ventricle; LA: left atrium; LMCA: left main coronary artery; LAD : left anterior descending coronary artery, CX: circumflex coronary artery. 
In Kawasaki-like disease patients, changes in coronary artery are characterized by increased brightness and parietal thickening of the coronary arteries in echocardiography, and an increase in the z-score, although giant aneurysms are rarely observed. In several series published during the pandemic, coronary involvement in MIS-C occurred from 8 to $36 \%$, probably due to endothelial dysfunction associated with cytokine storm caused by SARS-CoV- 2.

Pignatelli et al., ${ }^{11}$ in a multicenter study in Latin American countries observed that patients with MIS-C and cardiac involvement had more laboratory abnormalities, respiratory involvement, and more intensive care unit admissions. ${ }^{11}$

In addition, classic Kawasaki disease usually affects children up to five years of age (average age of three years), while Kawasaki-like disease occurs in older children (average age of nine years). This was well demonstrated by Belay et al., ${ }^{12}$ in a study with 1,733 patients with MIS-C and median age of nine years (5-13 years); $66.5 \%$ of these patients had abdominal pain, $53.7 \%$ diarrhea, $55.6 \%$ skin rash, $53.6 \%$ conjunctival hyperemia, $50.8 \%$ arterial hypotension, $36.8 \%$ shock, $31 \%$ myocardial dysfunction, $17.3 \%$ myocarditis and $16.5 \%$ coronary dilation. It is worth remembering that myocarditis, ventricular dysfunction, shock and the need for intensive care in classic Kawasaki disease are very rare. ${ }^{12}$

Diniz et al., ${ }^{13}$ evaluated an interesting correlation between echocardiographic abnormalities, biomarkers of inflammation and myocardial injury in 48 patients with MIS-C. ${ }^{13}$ There was a statistically significant correlation between left ventricular dysfunction and increased D-dimer, C-reactive protein, ferritin and troponin; there was also a significant correlation between right ventricular dysfunction and increased D-dimer and C-reactive protein. Coronary abnormalities correlated only with D-dimer elevation.

The Clinical Guidance for Pediatric Patients with MIS-C recommends, for patients with MIS-C, SARS-CoV-2 infection and hyperinflammation, measurement of $\mathrm{BNP} /$ troponin at diagnosis, and serial controls until discharge, and ECG during and after hospitalization. Also, performance of echocardiography to aid in the diagnosis (with evaluation of ventricular function, valvular alterations, pericardial effusion and the coronary arteries), as well as in adequate treatment and subsequent follow-up.

IVIG has been widely used for a variety of conditions including Kawasaki disease and toxic shock syndrome, as an effective and safe therapy. Although the use of IVIG has been extended to MIS-C and Kawasaki-like disease, its effectiveness still needs to be determined in these cases. Also, the exact mechanism of this therapy is not yet clear, but presumably, IVIG reduces the action of monocytes, macrophages and other cells, preventing the progression of myocardial injury. Belhadjer et al., ${ }^{14}$ detected an improvement in ventricular function in 25 of 35 MIS-C patients treated with IVIG. Corticosteroid therapy (methylprednisolone) also appears to be beneficial in preventing or attenuating the effects of inflammatory responses induced by SARS-CoV-2. ${ }^{14}$ The combination of IVIG and corticosteroid treatment seems to avoid major complications such as ventricular dysfunction and the need for more vasoactive drugs for hemodynamic support. The use of anticoagulants and antiplatelet agents should be considered in patients with MIS-C admitted to the intensive care unit, with risk of thromboembolism and significant elevations in D-dimer. ${ }^{15}$ Patients with Kawasaki-like disease and coronary aneurysms should use antiplatelet agents such as aspirin, and in some cases, the association with clopidogrel may be necessary.

With the increase in vaccination coverage of the general population, there has been a substantial reduction in deaths and hospitalizations. Vaccination of adolescents aged 12 and over is already becoming a reality in our country. This strategy will serve not only to protect children and adolescents from serious disease, but also to help control the transmission of the virus throughout the community. In addition, with the increase in vaccination rates, it is totally feasible to return to school, which is so fundamental for the physical, mental, intellectual and psychological well-being of our children and adolescents, who have been seriously harmed in many aspects in this pandemic. 


\section{References}

1. World Health Organization.(WHO). Weekly operational update on COVID-19-4 July 2021. Weekly operational update on COVID-195 July 2021.[Internet] [Cited in 2021 july 21] Available from: https://www.who.int/publications/m/item/weekly-operationalupdate-on-covid-19---5.

2. Rajapakse N, Dixit D. Human and novel coronavirus infections in children: a review. Paediatr Int Child Health. 2021;41(1):36-55.

3. World Health Organization.(WHO). Multisystem inflammatory syndrome in children and adolescents with COVID-19.[Internet].[Cited in 2021 july 12] Available from: https: //www.who.int/news-room/ commentaries/detail/multisystem-inflammatory-syndrome- in-childrenand-adolescents-withcovid- 19.

4. Henderson LA, Canna SW,. Friedman KG ,Gorelik M, Lapidus SK, Bassiri H, et al. American College of Rheumatology Clinical Guidance for Multisystem Inflammatory Syndrome in Children Associated With SARS-CoV-2 and Hyperinflammation in Pediatric COVID-19: Version 2. Arthritis Rheumatol.2021;73(4):e13-e29. DOI 10.1002/art.41616.

5. Sáfadi MA, Kfouri RA . Dados eipidemiológicos de Covid-19 em pediatria. São Paulo:Sociedade Brasileira de Pediatria: Departamento Científico de Imunizações e Departamento Científico de Infectologia . São Paulo 2021 (nota técnica).

6. Jones VG, Mills M, Suarez D, Hogan CA, Yeh D, Segal JB, et al. COVID-19 and Kawasaki Disease: Novel Virus and Novel Case. Hosp Pediatr. 2020. 10(6):537-40. doi:10.1542/hpeds.2020-0123.

7. DeBiasi R, Song X, Delaney M, Bell M, Smith K, Pershad J, et al. Severe COVID-19 in Children and Young Adults in the Washington, DC Metropolitan Region. J Pediatr. 2020;223:199-203. doi: 10.1016/j. jpeds.2020.05.007.
8. Verdoni L, Mazza A, Gervasoni A, Martelli L, Ruggeri M, Ciuffreda $\mathrm{M}$, et al. An outbreak of severe Kawasaki-like disease at the Italian epicentre of the SARS-CoV-2 epidemic: an observational cohort study. Lancet.2020;395(10239):1771-8. doi:10.1016/S0140-6736(20)31103-X.

9. Chara BS, Rickli JM, Silva CF. Kawasaki-like disease, a new phenotype in Sars - CoV-2 ? Int J Cardiovasc Sci. 2021; 34(5):566-569. doi: https://doi.org/10.36660/ijcs.20200186.

10. Feldstein LR, Rose EB, Horwitz SM,Collins JP, Newhams MM, Son MBF, et al. Multisystem Inflammatory Syndrome in U.S. Children and Adolescents. N Engl J Med.2020;338(4):334-6.

11. Pediatric multisystem SARS COV2 with versus without cardiac involvement: a multicenter study from Latin America. Pignatelli R, Vazquez CA, Rivera IR. Eur J Pediatr.2021;180(9):2879-88. https://doi. org/10.1007/s00431-021-04052-9.

12. Belay ED, Abrams J, Oster ME, Giovanni J, Pierce T, Meng L, et al. Trends in geographic and temporal distribution of US children with multisystem inflammatory syndrome during the COVID-19 pandemic. JAMA Pediatr. 2021;175(8):837-45. doi:10.1001/jamapediatrics.2021. 0630.

13. Diniz MFR, Cardoso MF, Sawamura KSS. The Heart of Pediatric Patients with COVID-19: New Insights from a Systematic Echocardiographic Study in a Tertiary Hospital in Brazil. Arq Bras Cardiol. Ju 182021. S006-782X2021005008204. Doi: 10.36660/abc.2020920.

14. Belhadjer Z, Meot M, BajolleF, Khraiche D, Legendre A, Abakka S, et al. Acute heart failure in multisystem inflammatory syndrome in children (MIS-C) in the context of global SARS-CoV-2 pandemic. Circulation 2020;142:429-36.

15. Bansal N, Azeka E, Neunert C. Multisystem Inflammatory Syndrome Associated with COVID-19 Anti-thrombosis Guideline of Care for Children by Action. Pediatr Cardiol. 2021 Jun 02;1-5 https://doi.org/10.1007/ s00246-021-02651-9. 